\title{
New accurate measurements of neutron emission probabilities for relevant fission products
}

\author{
J. Agramunt ${ }^{1, \text { a }}$, J.L. Tain ${ }^{1}$, F. Albiol ${ }^{1}$, A. Algora ${ }^{1,9}$, R. Caballero-Folch ${ }^{2,3}$, F. Calviño ${ }^{2}$, G. Cortes ${ }^{2}$, I. Dillmann ${ }^{3}$, \\ A. Riego ${ }^{2}$, B. Rubio ${ }^{1}$, A. Saastamoinen ${ }^{4}$, P. Salvador-Castiñeira ${ }^{2}$, A. Tarifeño-Saldivia ${ }^{2}$, A. Tolosa ${ }^{1}$, and E. Valencia ${ }^{1}$ \\ 1 Instituto de Fisica Corpuscular, CSIC - Universidad de Valencia, 46980 Paterna, Spain \\ 2 Secció d'Enginyeria Nuclear, Universitat Politecnica de Catalunya, 08028 Barcelona, Spain \\ 3 TRIUMF, Vancouver, British Columbia V6T2A3, Canada \\ ${ }^{4}$ University of Jyvaskyla, Department of Physics, PO Box 35, 40014 University of Jyvaskyla, Finland \\ 5 GSI Helmholtzzentrum für Schwerionenforschung GmbH, 64291 Darmstadt, Germany \\ ${ }^{6}$ Department of Physics, University of Surrey, Guildford GU2 7XH, UK \\ 7 Department of Physics, University of Istanbul, 34134 Vezneciler, Turkey \\ 8 Centro de Investigaciones Energéticas Medioambientales y Tecnológicas, 28040 Madrid, Spain \\ 9 Institute of Nuclear Research of the Hungarian Academy of Sciences, 4001 Debrecen, Hungary
} T. Eronen ${ }^{4}$, A.R. Garcia ${ }^{8}$, E. Ganioglu ${ }^{7}$, W. Gelletly ${ }^{6}$, D. Gorelov ${ }^{4}$, V. Guadilla ${ }^{1}$, H. Hakala ${ }^{4}$, A. Jokinen ${ }^{4}$, A. Kankainen $^{4}$, A. Montaner ${ }^{1}$, M. Marta ${ }^{5}$, E. Mendoza ${ }^{8}$, I. Moore ${ }^{4}$, C. Nobs ${ }^{6}$, S. Orrigo ${ }^{1}$, H. Penttila ${ }^{4}$, M. Reponen ${ }^{4}$, S. Rinta-Antila ${ }^{4}$,

\begin{abstract}
We have performed new accurate measurements of the beta-delayed neutron emission probability for ten isotopes of the elements $\mathrm{Y}, \mathrm{Sb}, \mathrm{Te}$ and I. These are fission products that either have a significant contribution to the fraction of delayed neutrons in reactors or are relatively close to the path of the astrophysical $r$ process. The measurements were performed with isotopically pure radioactive beams using a constant and high efficiency neutron counter and a low noise beta detector. Preliminary results are presented for six of the isotopes and compared with previous measurements and theoretical calculations.
\end{abstract}

\section{Introduction}

Beta delayed neutron emission occurs for neutron-rich nuclei when the neutron separation energy in the daughter nucleus $S_{n}$ becomes smaller than the energy window for the decay $Q_{\beta}$. For decays proceeding to excited levels above $S_{n}$, neutron emission competes efficiently with the electromagnetic de-excitation. Under the common assumption that $\Gamma_{\gamma} / \Gamma_{n} \approx 0$ the probability of neutron emission $P_{n}$ is just the integral of the $\beta$-intensity distribution $I_{\beta}\left(E_{x}\right)$ in the interval $\left[S_{n}, Q_{\beta}\right]$. Therefore the rate of delayed neutron emission is sensitive to nuclear structure. In general as the neutron excess increases so does $P_{n}$ until eventually it becomes the dominant decay mode.

Delayed neutrons are produced in nuclear power reactors in the decay of some fission products. Although they are a small fraction (less than one percent) of the total neutron inventory, they play a dominant role in reactor control. The effective delayed neutron fraction $\beta_{\text {eff }}$, modulates the change of reactor power to sudden changes of the reactor reactivity coefficient $\rho$ (which measures the departure from criticality [1]). This allows inter alia the safe insertion and extraction of absorption control rods. The delayed neutron fraction $\beta_{\text {eff }}$ is thus an important reactor parameter which must be known with good accuracy. This parameter is obtained from measurements of specific fissioning systems (integral measurements). It

a e-mail: agramunt@ific.uv.es can be obtained also from summation calculations which require the knowledge of fission yields $Y$ and $\beta$-delayed neutron emission probabilities $P_{n}$ of individual precursors. The advantage of the latter method is that it can be applied to systems for which no integral data exists. However summation calculations do not attain currently the level of accuracy necessary to replace the conventional method. Although this is mainly due to uncertainties in the yields $Y$, the improvement of the current knowledge of $P_{n}$ values for selected isotopes can have an impact on the calculations. In addition, from comparison of summation calculations and integral measurements, constraints on uncertain fission yields could be established. Currently, the discrepancies between calculated $\beta_{\text {eff }}$ and integral measurements (up to $10 \%$ ) result in an undesirable conservatism in the design and operation of reactor control systems [2]. Moreover new requirements are emerging in reactor technology because of the use of high burn-up fuels or the burning of minor actinides where the fission product inventory differs from that of conventional reactors. Thus improved measurements on delayed neutron emission probabilities for selected isotopes will contribute to the reduction in the uncertainties in this area.

Delayed neutrons also play a role in the synthesis of elements heavier than iron in the Universe through the astrophysical $r$ process. The $r$ process is associated with the occurrence of huge instantaneous fluxes of neutrons which through a succession of rapid neutron captures and $\beta$ decays builds up very neutron-rich nuclei up to the heaviest possible elements. The nuclei along this so called 
$r$ process pathway will decay towards the line of stability by a series of $\beta$ decays, accompanied by the emission of delayed neutrons in the case of the most neutron-rich isotopes. The delayed neutron emission process alters the mass distribution created initially, by shifting the decay path to lower masses on the one hand, and by providing additional neutrons for late captures on the other hand, which smooth and shift the final distribution to higher masses [3]. Therefore a knowledge of $P_{n}$, particularly for the most exotic nuclei, is an important ingredient in the calculation of the abundance of the elements either because they have a direct use or because they help to improve betastrength based $P_{n}$ theoretical estimations for those isotopes that are out of experimental reach.

We mentioned before that the value of $P_{n}$ is sensitive to the structure of the daughter and parent nuclei and therefore its measurement is also a tool in nuclear structure investigation. But this, in turn, is also important in the context of calculations of the $r$ process, which often have to rely on theoretical models to obtain the decay properties, including the half-life $T_{1 / 2}$ of many of the nuclei involved, which are not yet accessible to experimentation. The predictive power of these models can be improved if they are confronted with both $T_{1 / 2}$ and $P_{n}$ values sensitive to different parts of the $\beta$-intensity distribution.

We have performed a sensitivity study to select precursors that contribute appreciably to the delayed neutron fraction in reactors and have a relatively uncertain $P_{n}$ value, that are candidates for improved measurements. The selection was completed with isotopes that are relatively close to the astrophysical $r$ process path. In a previous measurement [4] we studied a number of isotopes from this list. Now a new measurement has been performed and data was taken for a total of ten isotopes of the elements $\mathrm{Y}, \mathrm{Sb}, \mathrm{Te}$ and I. The measurement and the analysis method will be described and preliminary results for six of the isotopes will be presented and discussed.

\section{Experimental apparatus}

Measurements were performed at the IGISOL-IV mass separator [5] installed at the Cyclotron Laboratory of the University of Jyväskylä (JYFL) producing a broad range of isotopes from proton-induced $\mathrm{E}_{p}=25 \mathrm{MeV}$ fission of a $U$ target inside the ion source. After mass separation of the ion beam with a moderate mass resolving power, the isotope of interest is isolated from accompanying isobars by means of a double Penning trap system [6]. The extracted beam shows high isotope purity provided that the isobars have a large mass difference with respect to the selected isotope in comparison to the trap frequency width, which was the case. The beam is implanted on a movable tape after traveling inside a $1 \mathrm{~mm}$ thick aluminum vacuum tube with a diameter of $46 \mathrm{~mm}$. The beam gate is open for a time length of three half-lives. Data acquisition starts 1-2 seconds before the gate is open and lasts for a total of ten half-lives. The tape we used is a standard half-inch computer tape with the magnetic layer facing the beam. We mounted on the tube end-cap a $3 \mathrm{~mm}$ thick plastic scintillation detector placed $6 \mathrm{~mm}$ behind the tape in order to count $\beta$ particles emitted by the implanted ion.

The beam tube goes through the central hole of the neutron counter reaching the center of the detector. The version of the neutron counter BELEN [7] used in this

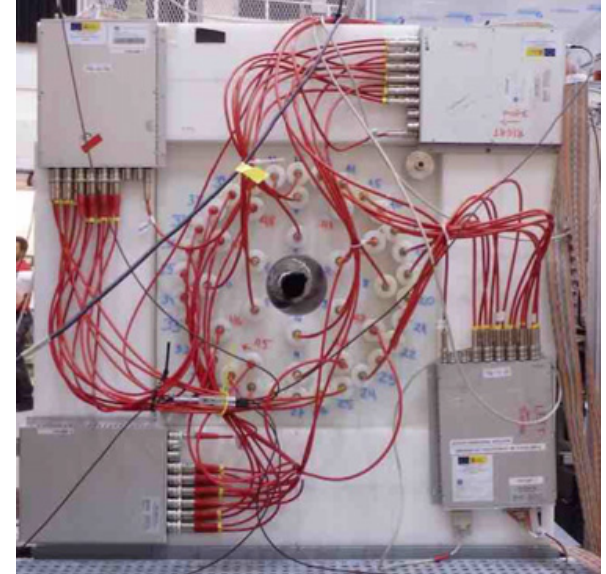

Figure 1. Front view of the BELEN-48 detector showing the disposition of the tubes in three rings around the central hole. The tubes are connected to the preamplifiers. The beam tube is inserted from the back. A HPGe detector (not shown) is inserted at the front.

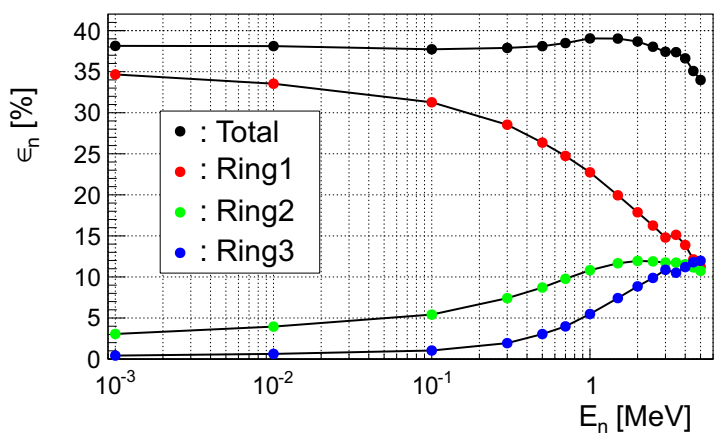

Figure 2. Neutron efficiency as a function of energy simulated with MCNPX. The contribution of each ring of tubes is shown.

measurement consists of forty eight ${ }^{3} \mathrm{He}$ proportional tubes arranged in three concentric rings inside a large polyethylene block (see Fig. 1). The rings have six, twelve and thirty tubes respectively. The central hole has a diameter of $11 \mathrm{~cm}$ able to accommodate a large HPGe detector for $\gamma$-ray counting. The spatial disposition of the tubes was optimized using MCNPX [8] Monte Carlo simulations in order to maximize the total detection efficiency having at the same time a nearly constant value up to $3 \mathrm{MeV}$ (see Fig. 2). The 1-inch tubes were fabricated by LND Inc. [9] and have pressures of 8 or 10 atmospheres.

The $\beta$ plastic detector is optically coupled with a Plexiglas light guide to a segmented photomultiplier tube R7600U-M4 from Hamamatsu. The signals coming from each half of the PMT anode are connected to a coincidence unit that cancels out the noise of individual segments and allows to set a low threshold $(50 \mathrm{keV})$. The signals from the ${ }^{3} \mathrm{He}$ tubes are fed to four MPR-16-HV charge sensitive preamplifiers modules from Mesytec. The differential output signals from the MPR-16-HV are converted into single-ended with a home-made converter (designed at JYFL), and plugged to the SIS3316 [10] sampling ADC of the Gasific data acquisition system where each channel is running in self-triggered mode registering time-stamp and signal amplitude for each detected neutron [7]. 
Table 1. $P_{n}$ values obtained in this work, compared to previous evaluations and theoretical calculations. The coincidence rate method, Eq. (2), is used for Te isotopes while total rates, Eq. (1), are used for the rest.

\begin{tabular}{lcccccc}
\hline Isotope & This work & PKM [12] & RAS [11] & FRDM-QRPA [13] & KTUY-GT2 [14] & RHB-RQRPA [15] \\
\hline${ }^{135} \mathrm{Sb}$ & $24.5(10)$ & $22.0(27)$ & $21.0(11)$ & 31.7 & 28.0 & 4.4 \\
${ }^{138} \mathrm{I}$ & $4.98(18)$ & $5.17(36)$ & $5.56(22)$ & 2.4 & 3.6 & 0.6 \\
${ }^{139} \mathrm{I}$ & $9.27(33)$ & $10.8(12)$ & $10.3(4)$ & 22.8 & 10.5 & 1.5 \\
${ }^{140} \mathrm{I}$ & $7.60(28)$ & $14.4(63)$ & $9.2(6)$ & 32.5 & 10.2 & 1.3 \\
${ }^{137} \mathrm{Te}$ & $2.60(30)$ & $2.86(24)$ & $3.04(16)$ & 5.1 & 0.41 & 0.4 \\
${ }^{138} \mathrm{Te}$ & $4.80(23)$ & $6.3(21)$ & $6.3(21)$ & 1.5 & 0.8 & 0.6 \\
\hline
\end{tabular}

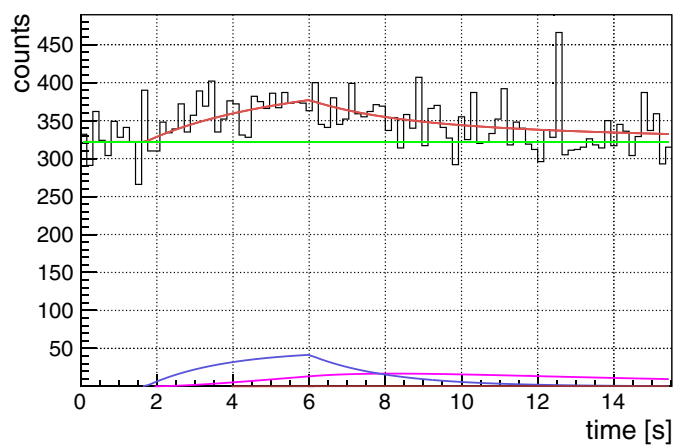

Figure 3. Time evolution of the neutron rate for ${ }^{138} \mathrm{Te}$ and the fit including the contributions of all descendants and a constant background.

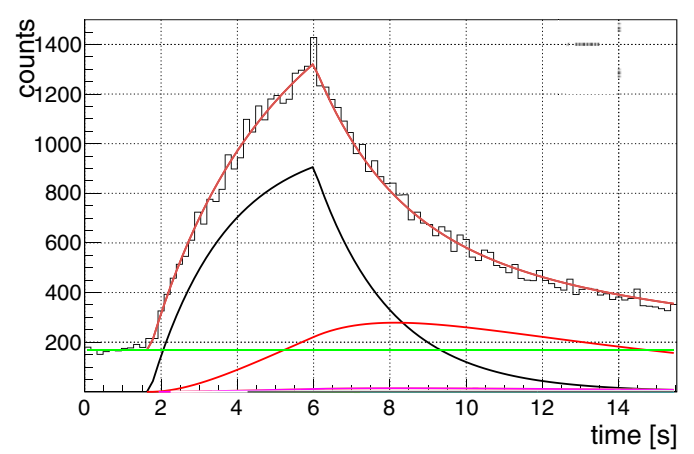

Figure 4. Time evolution of the $\beta$ rate for ${ }^{138} \mathrm{Te}$ and the fit including the contributions of all descendants and a constant background.

\section{Determination of $\boldsymbol{P}_{n}$ values}

To determine $P_{n}$ we use two different approaches depending on the collected neutron statistics. In one case we use the ratio of neutron counts to the number of $\beta$ counts (Eq. (1)) while in the other case we use the ratio of neutron- $\beta$ correlations to the number of $\beta$ particles (Eq. (2)), in both cases corrected by efficiencies [7]

$$
\begin{gathered}
P_{n}=\frac{\bar{\varepsilon}_{\beta}}{\bar{\varepsilon}_{n}} \frac{N_{n}}{N_{\beta}} \\
P_{n}=\frac{\bar{\varepsilon}_{\beta}}{\bar{\varepsilon}_{\beta}^{\prime}} \bar{\varepsilon}_{n} \frac{N_{\beta n}}{N_{\beta}}
\end{gathered}
$$

The advantage of Eq. (2) is that it can be applied for a very few number of detected decays because the $\beta$-neutron coincidence condition effectively suppress the neutron background. A disadvantage of this approach is the need to determine the average $\beta$ efficiencies for all decays $\left(\bar{\varepsilon}_{\beta}\right)$

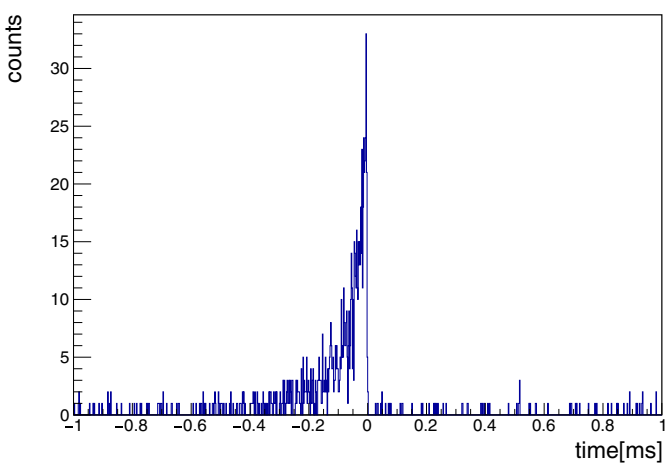

Figure 5. Time difference between each detected $\beta$ and all detected neutrons in the range from $-1 \mathrm{~ms}$ to $+1 \mathrm{~ms}$ for ${ }^{138} \mathrm{Te}$. The distribution reflects the neutron moderation time in the detector.

and for decays to states above $S_{n}\left(\bar{\varepsilon}_{\beta}^{\prime}\right)$. However for decays with a large $Q_{\beta n}=Q_{\beta}-S_{n}$ window both efficiencies will be very similar [7] and they will cancel out.

In the case of Eq. (1) in order to obtain $N_{n}$ and $N_{\beta}$ we fit simultaneously the time evolution of the neutron counting rate (Fig. 3) and the $\beta$ counting rate (Fig. 4). The value of the efficiency ratio $\bar{\varepsilon}_{\beta} / \bar{\varepsilon}_{n}=0.816(33)$ was obtained from ${ }^{95} \mathrm{Rb}$, a well know nucleus. This ratio shows little sensitivity to $Q_{\beta}$ and $S_{n}$ [7] thus can be applied to the remaining isotopes. Since the fit function depends on $P_{n}$ itself, we use an iterative procedure to obtain the value. The fit function corresponds to the appropriate solution of the Bateman equation and allows disentangling the contributions of the precursor and its descendants. This method is applied to obtain $P_{n}$ values for ${ }^{135} \mathrm{Sb}$ and $138,139,140$ I. For the iodine isotopes the fit function includes a loss factor for the daughter isotopes (xenon) which can escape rapidly from the implantation tape in a similar fashion as we did before for ${ }^{137}$ I [7].

In the case of Eq. (2) we assume cancellation of $\beta$ efficiencies and determine $N_{\beta n}$ from the time correlation distribution (Fig. 5) after background subtraction (determined from the positive part of the plot). We use a neutron efficiency $\bar{\varepsilon}_{n}=38.5(16) \%$, determined experimentally from ${ }^{95} \mathrm{Rb}$, that agrees well with the Monte Carlo simulation (see Fig. 2). The advantage of the $\beta-n$ method to extract $P_{n}$ in the case of ${ }^{138} \mathrm{Te}$ becomes apparent when comparing Fig. 3 with Fig. 5. For similar reasons it was also applied to ${ }^{137} \mathrm{Te}$ which has even lower statistics.

\section{Results and discussion}

The preliminary $P_{n}$ values obtained in this work are given in Table 1 and in graphical form in Fig. 6. These are compared with the result of previous evaluations 


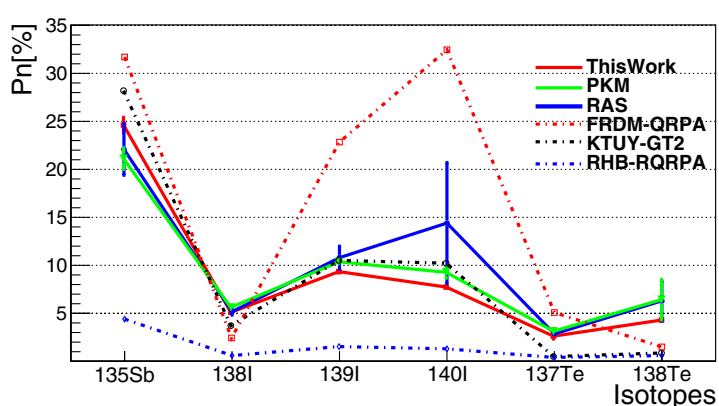

Figure 6. Results of this work, compared to previous evaluations and theoretical calculations.

by Rudstam et al. (RAS) [11] and by Pfeiffer et al. (PKM) [12] as well as with three theoretical calculations. The calculations are those from Moeller et al. (FRDMQRPA) [13], Koura et al. (KTUY-GT2) [14] and Marketin et al. (RHB-RQRPA) [15]. These are global calculations, covering the whole nuclide chart, suited for $r$ process abundance calculations.

Compared to previous evaluations (RAS and PKM) our results are slightly larger $(10-15 \%)$ for ${ }^{135} \mathrm{Sb}$ and slightly lower $(5-15 \%)$ for ${ }^{138} \mathrm{I}$ and ${ }^{139} \mathrm{I}$. For ${ }^{140} \mathrm{I}$ the PKM evaluated value has a large uncertainty (44\%) and is $56 \%$ larger than the RAS evaluation, which has a $7 \%$ uncertainty. Our result is $17 \%$ lower than the value in RAS. For these four isotopes the relative uncertainty (statistical) of our results is about $4 \%$. In the case of ${ }^{137} \mathrm{Te}$ our result is more uncertain $(12 \%)$ and is slightly lower (10-15\%) than both evaluations. In the case of ${ }^{138} \mathrm{Te}$ there exist a single previous measurement [16] with a large uncertainty (33\%) quoted in both evaluations. Our result has an uncertainty (statistical) of $5 \%$ and is $25 \%$ smaller than the previous value. Although with marginal statistics (see Fig. 3) the $P_{n}$ value obtained using Eq. (1) agrees well with the quoted value obtained with Eq. (2). The half-life for ${ }^{138} \mathrm{Te} T_{1 / 2}=$ 1.4(4) s has also a relatively large uncertainty. In our fit this half-life was left as a free parameter obtaining in this way an improved estimate of $T_{1 / 2}=1.412(18) \mathrm{s}$.

Comparing the theoretical calculations to the data one observes (Fig. 6) that all three models reproduce the isotopic trend (position of maxima and minima). However the RHB-RQRPA values are strongly suppressed (by a factor of 5 to 8 ) with respect to the experiment. The FRDM-QRPA model agrees with experiment within a factor of 2 for ${ }^{135} \mathrm{Sb},{ }^{138} \mathrm{I}$ and ${ }^{137} \mathrm{Te}$, overpredicts the values of ${ }^{139,140} \mathrm{I}$ by a factor of 3-4 and underpredicts the value of ${ }^{138} \mathrm{Te}$ by a factor of 3 . The KTUY-GT2 model predicts fairly well the $P_{n}$ values of the antimony and iodine isotopes but underpredict the values for the tellurium isotopes by a factor of 6 .

\section{Conclusion}

We have obtained accurate $P_{n}$ values for ${ }^{135} \mathrm{Sb},{ }^{138,139,140} \mathrm{I}$ and ${ }^{137,138} \mathrm{Te}$. In particular for ${ }^{140} \mathrm{I}$ and ${ }^{138} \mathrm{Te}$ our data represent a significant improvement with respect to previous values. We found that the theoretical calculations of Ref. [15] grossly underpredict all the experimental values. The calculations of Ref. [13] differ significantly from experiment for ${ }^{139,140} \mathrm{I}$ and ${ }^{138} \mathrm{Te}$. The calculations of Ref. [14] agree well with experiment except for the tellurium isotopes.

This work was supported by Spanish Ministerio de Economia y Competitividad under grant numbers FPA2011-24553, FPA 2011-28770-C03-03, FPA2014-52823-C2-1-P/-2-P, CPAN CSD2007-00042 (Ingenio2010) and the program Severo Ochoa (SEV2014-0398). Work supported by the European Commission under the FP7/EURATOM contract 605203 (CHANDA). Work supported by the Finnish Centre of Excellence Programme 2012-2017 (Project No. 21350). Work partially done within IAEA-CRP for Beta Delayed Neutron Data.

\section{References}

[1] S. Glasstone and A. Sesonske, Nuclear Reactor Engineering, Krieger, 1981

[2] A. DAngelo, Prog. Nucl. Ener. 41, 5 (2002)

[3] A. Arcones et al., Phys. Rev. C 83, 045809 (2011)

[4] J. Agramunt et al., Nucl. Data Sheets 120, 74 (2014)

[5] I. Moore et al., Nucl. Instrum. Methods Phys. Res., Sect. B 317, 208 (2013)

[6] T. Eronen et al., Eur. Phys. J. A 48, 46 (2012)

[7] J. Agramunt, et al., Nucl. Instrum. Methods Phys. Res., Sect. A 807, 69 (2016)

[8] D. Pelowitz (E.), MCNPX Users Manual, Los Alamos, Report LA-CP-05-0369, 2005

[9] LND, INC. http: //www. Indinc. com/

[10] Struck Innovative Systeme. http://www.struck. de/

[11] Rudstam et al., Atom. Data Nucl. Data Tables 53, 1 (1993)

[12] Pfeiffer et al., Prog. Nucl. Energy 41, 39 (2002)

[13] Moeller et al., Phys. Rev. C 67, 55802 (2003)

[14] H. Koura et al., Prog. Theor. Phys. 113, 305 (2005)

[15] T. Marketin et al., Phys. Rev. C 93, 025805 (2016)

[16] M. Ashgar et al., Nucl. Phys. A 247, 359 (1975) 